\title{
Effects of feeding an immunomodulatory supplement to heat-stressed or actively cooled cows during late gestation on postnatal immunity, health, and growth of calves
}

\author{
Amy L. Skibiel, ${ }^{*}$ Thiago F. Fabris, ${ }^{*}$ Fabiana N. Corrá, ${ }^{*}$ Yazielis M. Torres, ${ }^{*}$ Derek J. McLean, $\dagger$ \\ James D. Chapman, † David J. Kirk, † Geoffrey E. Dahl,* and Jimena Laporta*1 \\ *Department of Animal Sciences, University of Florida, Gainesville 32611 \\ †Phibro Animal Health Corporation, Teaneck, NJ 07666-6712
}

\section{ABSTRACT}

Heat stress during late gestation negatively affects the physiology, health, and productivity of dairy cows as well as the calves developing in utero. Providing cows with active cooling devices, such as fans and soakers, and supplementing cows with an immunomodulating feed additive, OmniGen-AF (OG; Phibro Animal Health Corporation), improves immune function and milk yield of cows. It is unknown if maternal supplementation of OG combined with active cooling during late gestation might benefit the developing calf as well. Herein we evaluated markers of innate immune function, including immune cell counts, acute phase proteins, and neutrophil function, of calves born to multiparous dams in a $2 \times 2$ factorial design. Dams were supplemented with OG or a bentonite control (NO) beginning at $60 \mathrm{~d}$ before dry off and exposed to heat stress with cooling (CL) or without active cooling (HT) during the dry period $(\sim 46 \mathrm{~d})$. At birth, calves were separated from their dams and fed $6.6 \mathrm{~L}$ of their dams' colostrum in 2 meals. Calf body weight and rectal temperature were recorded, and blood samples were collected at birth (before colostrum feeding) and at 10, 28 , and $49 \mathrm{~d}$ of age. Calves born to either CL dams or OG dams were heavier at birth than calves born to HT or NO dams, respectively. Concentrations of serum amyloid A were higher in the blood of calves born to OG dams relative to $\mathrm{NO}$ and for $\mathrm{HT}$ calves relative to CL calves. In addition, calves born to cooled OG dams had greater concentrations of plasma haptoglobin than calves born to cooled control dams. Neutrophil function at $10 \mathrm{~d}$ of age was enhanced in calves born to cooled OG dams and lymphocyte counts were higher in calves born to OG dams. Together these results suggest that adding OG to maternal feed in combination with ac-

Received January 19, 2017.

Accepted May 7, 2017.

${ }^{1}$ Corresponding author: jlaporta@ufl.edu tive cooling of cows during late gestation is effective in mitigating the negative effects of in utero heat stress on postnatal calf growth and immune competence.

Key words: feed supplement, acute phase protein, hematology, neutrophil

\section{INTRODUCTION}

Exposure of dairy cows to heat stress during the dry period, 6 to 8 wk before calving, reduces milk production in the following lactation (Tao et al., 2011), alters metabolism, and compromises both the innate and adaptive immune function of the cow (do Amaral et al., 2009, 2010, 2011; Tao et al., 2012b). Heat stress not only adversely affects the cow, but recent research also points to short- and long-term consequences of maternal heat stress during late gestation on the calf in utero. Calves born to heat-stressed cows are lighter at birth, and this difference in weight continues through puberty (Monteiro et al., 2016a). Heifers born to heat stressed cows have lower fertility and produce less milk, at a rate of $5 \mathrm{~kg} / \mathrm{d}$ through $35 \mathrm{wk}$ of their first lactation relative to in utero-cooled heifers (Monteiro et al., 2016b). Moreover, in utero heat stress affects calf metabolism postnatally and induces alterations in immune development (Tao et al., 2014; Dahl et al., 2016; Guo et al., 2016; Monteiro et al., 2016a). Specifically, heat-stressed calves in utero have lower absorption of IgG from colostrum (Tao et al., 2012a; Monteiro et al., 2014; Laporta et al., 2017) and have lower rates of peripheral blood mononuclear cell (PBMC) proliferation, which persists to at least $56 \mathrm{~d}$ of age. (Tao et al., 2012a). Together, these studies indicate compromised passive immune transfer and adaptive immunity during early life of heat-stressed calves in utero.

Heat stress abatement, by providing fans and soakers during the dry period (late gestation), improves lactation performance, metabolism, and immune function of cows and their calves (Tao and Dahl, 2013; Dahl et al., 2016). Additionally, nutritional management practices 
may help to ameliorate some of the negative effects of heat stress on immune function and lactation performance. OmniGen-AF (OG), a patented proprietary feed additive (Phibro Animal Health Corporation, Teaneck, NJ), was developed to support immune function and has documented immunomodulatory benefits when supplemented to ruminants and rodent models (Wang et al., 2009; Rowson et al., 2011; Ryman et al., 2013; Branson et al., 2016). For example, supplementing dairy cows with OG from -35 to $46 \mathrm{~d}$ relative to calving increased daily milk yield and improved innate immune response of dairy cows when challenged with a pathogen (Brandão et al., 2016). Similarly, adding OG to the feed of immunosuppressed sheep increased concentrations of circulating neutrophils and lymphocytes and increased L-selectin on the neutrophil surface (Wang et al., 2007). OmniGen-AF contains a mixture of silicon dioxide, calcium aluminosilicate, sodium aluminosilicate, brewers dehydrated yeast, mineral oil, calcium carbonate, rice hulls, niacin supplement, biotin, D-calcium pantothenate, vitamin $\mathrm{B}_{12}$ supplement, choline chloride, thiamine mononitrate, pyridoxine hydrochloride, riboflavin-5-phosphate, and folic acid, but the full formulation is proprietary. Additionally, OG may be beneficial for heat stress abatement in dairy cows by decreasing respiration rate and rectal temperature during lactation (Hall et al., 2014) and the dry period (Fabris et al., 2017). Moreover, cytokine gene expression was upregulated in lactating heat-stressed cows fed OG, which may be associated with improved immune function (Hall et al., 2014). However, it is unknown whether supplementation of OG cows during late gestation may affect the offspring.

Our objective was to evaluate the effect of OG supplementation to pregnant cows exposed to heat stress with or without cooling during late gestation on growth and markers of immunity of preweaned calves. We hypothesized that feeding OG to heat-stressed dams during late gestation would improve their offspring's immune function and performance during the preweaning period. Active cooling of heat-stressed dams during late gestation combined with OG supplementation was predicted to further improve these responses in the offspring.

\section{MATERIALS AND METHODS}

\section{Animals and Experimental Design}

Maternal Treatments. We conducted a trial at the Dairy Unit of the University of Florida (Hague, FL) during summer and fall (June to December) of 2015. Treatments and animal handling were approved by the
University of Florida Institute of Food and Agricultural Sciences Animal Research Committee. Pregnant multiparous Holstein cows were randomly assigned to dietary treatments to receive OG supplementation (56 $\mathrm{g} / \mathrm{d}$ ) or no-OmniGen-AF supplementation (NO; $56 \mathrm{~g} / \mathrm{d}$ of bentonite as a placebo, AB20, Phibro Animal Health Corporation) at $60 \mathrm{~d}$ before dry off. Approximately 46 d before expected calving, cows within each dietary treatment were dried off and assigned (based on parity and mature equivalent milk production of their previous lactation) to heat stress (HT) or cooling (CL) treatments. Thus, cows were divided into 4 groups $(2 \times 2$ factorial design): cooled no OG (CLNO), no cooling no OG (HTNO), cooled OG (CLOG), and no cooling OG (HTOG). All cows were housed in the same freestall barn during the dry period, but CL cows were actively cooled using fans (J\&D Manufacturing, Eau Claire, WI), over the manger and freestalls, and water soakers (Rain Bird Manufacturing, Glendale, CA), over the manger; HT cows were only provided with shade. Fans were on at all times, whereas soakers turned on automatically for $1.5 \mathrm{~min}$ at 5 -min intervals when ambient temperature exceeded $21.1^{\circ} \mathrm{C}$. Ambient temperature and relative humidity of each barn were recorded every 15 min with Hobo Pro series Temp probes (Onset Computer Corp., Pocasset, MA). The temperature-humidity index (THI) was calculated for each barn based on the equation reported by Dikmen et al. (2008). Rectal temperature of the dams was measured twice daily $(0730$ and $1430 \mathrm{~h})$, and respiration rates were monitored thrice weekly $(1400 \mathrm{~h}$ ) for all cows during the dry period. All cows were fed a common TMR during the lactation phase $(18.5 \% \mathrm{CP}, 29.8 \% \mathrm{NDF}, 0.87 \% \mathrm{Ca}$, $0.35 \% \mathrm{P}, 0.54 \% \mathrm{Na}, 0.59 \% \mathrm{Cl}, 0.45 \% \mathrm{Mg}$, and $0.23 \%$ $\mathrm{S})$ and the entire dry period $(15.4 \% \mathrm{CP}, 34.5 \% \mathrm{NDF}$, $0.62 \% \mathrm{Ca}, 0.37 \% \mathrm{P}, 0.36 \% \mathrm{Na}, 0.4 \% \mathrm{Cl}, 0.3 \% \mathrm{Mg}$, and $0.2 \% \mathrm{~S})$. From $60 \mathrm{~d}$ before dry off through dry off the supplement was mixed with the ration, whereas during the dry period it was top-dressed once daily.

Calf Management. Calves used in the current study were born to dams either supplemented or not supplemented with OG (beginning at $60 \mathrm{~d}$ before dry off until calving) and heat-stressed or cooled during the final $46 \pm 3 \mathrm{~d}$ of gestation. Sixty calves were born from these dams, of which 32 were heifers $(\mathrm{n}=$ 8 CLNO, $\mathrm{n}=8$ HTNO, $\mathrm{n}=8$ HTOG, and $\mathrm{n}=8$ CLOG) that were followed until weaning (d 49). Four heifers, 2 from CLNO, 1 CLOG, and 1 HTOG treatment, were removed from the study due to injuries at the time of calving or due to lack of sufficient colostrum for feeding. All calves were born between July 14 and October 6. Day of birth was considered study d 0. At birth, all calves were separated from their dams and 
had their navel dipped with $2 \%$ iodine to prevent infection. Within $2 \mathrm{~h}$ after calving, dams were milked in the parlor or using a portable milking unit according to the Dairy Unit standard operating procedures to collect colostrum (Donovan et al., 2011). Calves were fed $6.6 \mathrm{~L}$ of fresh colostrum from their dam in 2 separate meals $(3.8 \mathrm{~L}$ fed within $4 \mathrm{~h}$ and $2.8 \mathrm{~L}$ within $12 \mathrm{~h}$ after birth) by bottle or by esophageal feeder when needed to ensure colostrum delivery. Depending on colostrum production and availability, 2 calves were fed frozen colostrum collected from a different dam in the same treatment group. After $1 \mathrm{~d}$ of age, heifer calves were transported to the Calf Unit, housed in individual pens, and provided pasteurized milk $(6 \mathrm{~L} / \mathrm{d}$ the first $21 \mathrm{~d}$ and $8 \mathrm{~L} / \mathrm{d}$ thereafter by bucket in 2 meals at 0600 and $1800 \mathrm{~h}$ ) and ad libitum grain and water until weaning at $49 \mathrm{~d}$. Calves were weaned by reducing milk allotment to $3 \mathrm{~L} / \mathrm{d}$ for $1 \mathrm{wk}$, at which time milk feeding ceased. All calves were managed under identical conditions. All vaccinations were based on the Dairy Unit standard operating procedures for calves (Donovan et al., 2011)

\section{Data, Sample Collection, and Laboratory Analyses}

Blood Collection. Shortly after birth (before the first colostrum feeding; $\mathrm{d}=0$ ), at $24 \mathrm{~h}$ (after colostrum feeding; $\mathrm{d}=1$ ), and on $\mathrm{d} 10$ and 28 after calving, blood from the jugular vein was collected using 2 Vacutainer tubes $(10 \mathrm{~mL}$, Becton Dickinson, Franklin Lakes, NJ) containing clot activator gel (placed at room temperature for $\sim 30 \mathrm{~min}$ ) or sodium-heparin anticoagulant (placed immediately on ice before processing). Serum and plasma were separated through centrifugation at $3,000 \times g$ for 20 min at $4^{\circ} \mathrm{C}$, aliquoted, and stored frozen $\left(-20^{\circ} \mathrm{C}\right)$ until time of assay.

Apparent Efficiency of Immunoglobulin Absorption. Apparent efficiency of absorption (\%) was estimated by measuring the IgG content in first-milking colostrum and calf serum at $24 \mathrm{~h}$ after colostrum ingestion using the single radial immunodiffusion test (Bovine IgG Test Kit; Triple J Farms, Bellingham, WA), described previously by Monteiro et al. (2014) for calves. Calculations were performed using a formula described by Quigley and Drewry (1998): [plasma IgG $(\mathrm{g} / \mathrm{L}) \times$ birth weight $(\mathrm{kg}) \times$ plasma volume $(\mathrm{L}) / \mathrm{IgG}$ fed $(\mathrm{g})] \times 100$.

Hematology Analysis. A 2-mL sample of whole blood was collected from the jugular vein of calves on d 0 and 1 after birth into vacutainer tubes containing EDTA anticoagulant. Whole blood samples were kept on ice and transported to the laboratory within $2 \mathrm{~h}$ of collection. Blood parameters, including total and differential leukocyte counts (neutrophils, lymphocytes, basophils, eosinophils), red blood cells (mature red blood cells, reticulocytes, and platelets), and hemoglobin were measured using an automated hematology analyzer (ProCyte Dx Hematology Analyzer; Idexx Laboratories, Westbrook, ME).

Neutrophil Function. Assessment of the percentages of neutrophils undergoing phagocytosis and oxidative burst activity was performed using dual-color flow cytometry (FACSort; Becton Dickinson Immunocytometry Systems, San Jose, CA), as described previously by Martinez et al. (2012). Briefly, $100 \mu \mathrm{L}$ of whole blood from each calf at $24 \mathrm{~h}$ and on d 10 and 28 were loaded with $10 \mu \mathrm{L}$ of a $50 \mu M$ dihydrorhodamine 123 (Sigma-Aldrich, St. Louis, MO) solution for $10 \mathrm{~min}$ at $37^{\circ} \mathrm{C}$ with continuous mixing. Aliquots of propidium iodide-labeled Escherichia coli were added to achieve a bacteria-to-neutrophil ratio of 40:1. Each sample had a negative and a positive control tube. Tubes were placed on ice to stop neutrophil phagocytosis and oxidative burst activities. An automated lysing system (Q-Prep Epics Immunology Workstation; Coulter Corp., Miami, FL) was used to add reagents. Hemolysis was achieved by the addition of $500 \mu \mathrm{L}$ of water to each tube and extracellular fluorescence was suppressed with the addition of $10 \mu \mathrm{L}$ of $0.4 \%$ trypan blue solution (Thermo Fisher Scientific, Waltham, MA). Fluorescence cytograms were based on a logarithmic amplification of the fluorescence signals of each cell as it passed through the 488-nm argon-ion laser beam for excitation at 15 $\mathrm{mW}$ (FACSort; Becton Dickinson Immunocytometry Systems, San Jose, CA). Neutrophils were analyzed on the basis of their size and complexity in the density cytogram. Data from the acquisition of 10,000 cells per sample were processed by CellQuest v3.3 (Becton Dickinson Immunocytometry Systems, San Jose, CA). Parameters quantified included the percentage of neutrophils that contained red fluorescence, indicating phagocytosis of propidium iodide-labeled E. coli, and the percentage of neutrophils with red and green fluorescence, indicating oxidative burst. The mean amount of reactive oxygen intermediates produced per neutrophil and the mean number of bacteria phagocytized by neutrophils were reported as the mean fluorescence intensity of the green and red wavelengths, respectively. Analysis was performed using the FlowJo v10.1 (Single Cell Analysis Software, LLC, Ashland, OR).

Haptoglobin and Serum Amyloid A. Plasma haptoglobin (HPT) was measured on d 0, 10, and 28 using a colorimetric procedure that measures HPThemoglobin complexing by estimating differences in peroxidase activity with results expressed as optical density, measured at $450 \mathrm{~nm}$ on a microplate reader. This protocol has been validated for bovine plasma 
samples previously (Cooke and Arthington, 2013). The absolute absorbance values were used for statistical analysis. Samples were analyzed in triplicate and the inter- and intra-assay coefficients of variation were 5.1 and $3.1 \%$, respectively. Concentrations of serum amyloid A (SAA) were measured using a commercial solid phase sandwich ELISA PHASE Serum Amyloid A Assay (Cat\# TP-802, Tri-Delta Diagnostics Inc., Morris Plains, NJ) following the manufacturer's protocol, with samples diluted 1:250 in 1× diluent buffer to fit within the standard curve of the assay. Samples were analyzed in duplicate, and the inter- and intra-assay coefficients of variation were 4.1 and $7.0 \%$, respectively.

Growth Parameters and Health. Heifer BW, hip height, and rectal temperature were obtained on d 0,10 , 28 , and at weaning (d 49). Heifer health was assessed on d 10, 28, and 49, with scores assigned according to the University of Wisconsin Calf Health Score Chart (McGuirk, 2008).

\section{Statistical Analysis}

Data were analyzed using SAS version 9.4 (SAS Institute, Cary, NC). Measurements at birth were analyzed using a generalized linear mixed model with calf sex, treatments, and their interaction as fixed effects, and calf identification as a random effect. Measurements over time were analyzed using general linear models including the fixed effect of treatments, day (repeated measure), and their interactions (excluding the effect of sex). The first-order autoregressive covariance structure AR (1) was used as the covariate structure. Values reported are least squares means \pm standard error of the mean. Normality and outlier tests were performed for all the variables analyzed. Blood HPT and SAA were $\log$-transformed due to lack of normality of the data. Differences with $P \leq 0.05$ were considered statistically significant and between $0.05 \leq P \leq 0.10$ tending toward significance.

\section{RESULTS}

\section{Indicators of Heat Stress During Late Gestation}

The effectiveness of the heat stress treatment applied during the dry period to the cows was confirmed. The average THI during the experimental period was similar between the HT and CL pens (77.6 and 77.9 for CL and HT, respectively) and was $\geq 68$ during the day and night for the entire period, indicating all cows were exposed to a similar degree of heat stress. The cooling system effectively reduced the heat strain of CL cows; rectal temperature was 39.3 vs. $38.9 \pm 0.03^{\circ} \mathrm{C}$, for HT and CL cows, respectively $(P<0.001)$, and HT cows had higher respiration rates $(73.5$ vs. $44.5 \pm 1.3$ breaths/min, for HT and CL cows, respectively; $P<$ 0.001). Cows supplemented with OG had lower respiration rates than NO cows during late gestation $(P<$ $0.01,69$ vs. $73 \pm 1.6$ breaths $/ \mathrm{min})$.

\section{BW and Rectal Temperature}

Calves born to HT cows were $3.5 \mathrm{~kg}$ lighter relative to calves born to CL cows ( 38.8 vs. $42.3 \pm 1.0 \mathrm{~kg}$, for HT and CL calves, respectively; $P<0.01$; Table 1 ). Calves born to OG-supplemented dams tended to be heavier at birth than those born to nonsupplemented dams (41.7 vs. $39.5 \pm 0.95 \mathrm{~kg}$, for $\mathrm{OG}$ and NO, respectively; $P=$ 0.06 ; Table 1). Gestation length was shortened by $4 \mathrm{~d}$ in HT cows compared with CL cows (272.8 vs. $274.4 \pm 1.4$ d, respectively), however, it was not affected by OG. We found no interaction between treatments. Heat stress during the dry period affected the rectal temperature of the dam at the time of calving, which was $0.5^{\circ} \mathrm{C}$ higher compared with CL dams $\left(39.5\right.$ vs. $39.0 \pm 0.1^{\circ} \mathrm{C} ; P<$ 0.01 ), but there was no effect of $\mathrm{OG}$ supplementation on dam's rectal temperature at calving. We observed an interaction between treatments for calf rectal temperature at birth (Table 1); calves born from HTNO dams tended to have higher rectal temperature at birth compared with the other groups $\left(39.5^{\circ} \mathrm{C}\right.$ for HTNO vs. $38.3^{\circ} \mathrm{C}$ average for CLNO, CLOG, and HTOG; $P<$ 0.08). In addition, during the preweaning period, heifers born to HT dams had higher rectal temperature relative to heifers born from CL dams (39.3 vs. 39.0 $\pm 0.06^{\circ} \mathrm{C}$, respectively; $P=0.01$; Table 1$)$. Similarly, heifers born from OG-supplemented dams tended to have lower rectal temperature than those born to nonsupplemented dams (39.0 vs. $39.2 \pm 0.0 .06^{\circ} \mathrm{C}$ for $\mathrm{OG}$ and NO, respectively; $P=0.10$; Table 1 ).

\section{Growth Performance and Health}

Average daily gain was similar from birth to d 10 and from birth to $\mathrm{d} 28$ for all groups of heifers and averaged $1.36 \pm 0.15 \mathrm{~kg} / \mathrm{d}$. However, heifers born from CL cows tended to gain $0.13 \mathrm{~kg}$ more per day from birth to weaning than those born to HT cows $(P=0.07$, Table 1). We found no effect of maternal OG supplementation on ADG of the heifers. All heifers were healthy through the experimental period evaluated. Although a few instances of minor nasal discharge were noted, there were no cases of ocular discharge and none of the health characteristics monitored throughout the preweaning period were significantly different between treatments (data not shown). 
Table 1. Body weight, rectal temperature (RT), hip height (HH), and ADG from calves born to cool (CL) or heat-stress (HT) cows supplemented (OG) or not supplemented (NO) with OmniGen-AF (Phibro Animal Health Corporation, Teaneck, NJ) during late gestation (dry period, approximately $46 \mathrm{~d}$ )

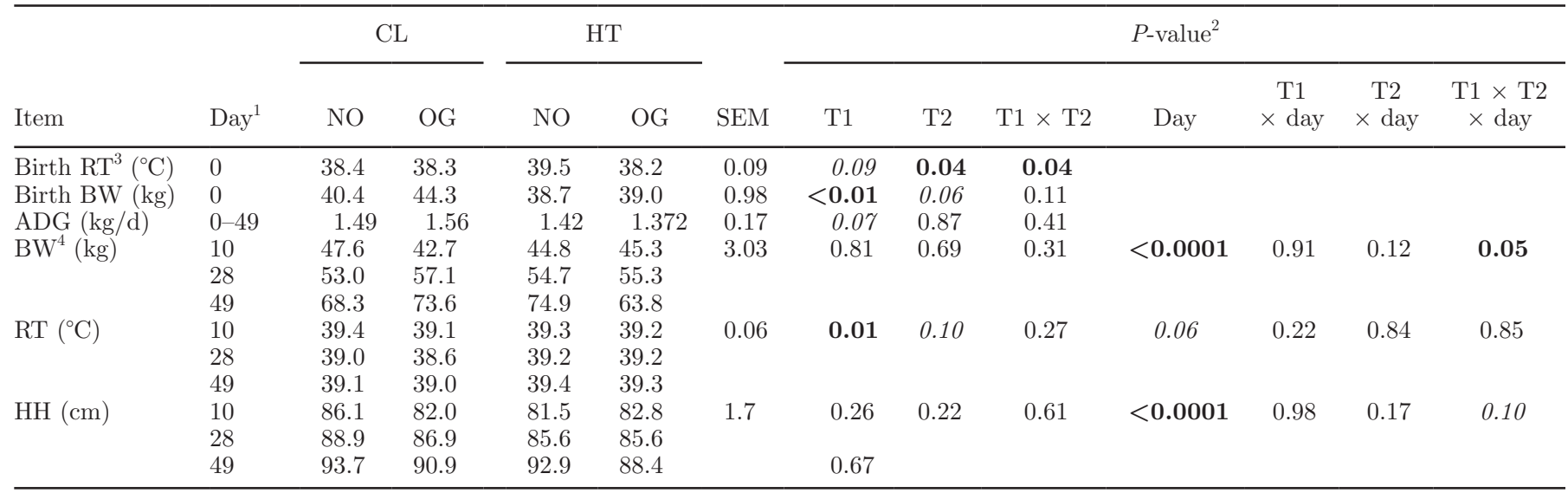

${ }^{1}$ Day 0 (birth), 10, 28, and 49 (weaning).

${ }^{2} \mathrm{~T} 1=$ heifers born from dams that were heat stressed $(\mathrm{HT})$ or cooled (CL) during late gestation; T2 = heifers born from dams supplemented with OmniGen-AF $(56 \mathrm{~g} / \mathrm{d})$ or not supplemented during late gestation (n $=6-8$ per treatment). $P \leq 0.05$ indicates statistical difference (in bold), and $0.05<P$-value $<0.10$ indicates a tendency (italics).

${ }^{3} \mathrm{LSM} \pm \mathrm{SEM}$ for the interaction between treatments.

${ }^{4} \mathrm{LSM} \pm \mathrm{SEM}$ for the interaction between treatments and day.

\section{Efficiency of IgG Absorption}

Dams' colostrum IgG concentration was higher for the HT compared with the CL cows (100 vs. $129 \pm 6.4$ $\mathrm{g} / \mathrm{L}$, respectively; $P<0.01$ ) and there was no effect of OG supplementation of the dam on colostrum IgG concentration (average $122 \pm 5.6 \mathrm{~g} / \mathrm{L}$ ). Colostrum volume was not evaluated in this study. Even though all calves achieved passive transfer at $24 \mathrm{~h}$, the apparent efficiency of IgG absorption was decreased in calves born to HT dams compared with those born to CL dams (19 vs. $13 \%$, respectively; $P=0.02$ ), but we found no overall effect of OG supplementation $(P=0.56)$.

\section{Acute Phase Proteins}

We noted a significant interaction between maternal OG supplementation and day for SAA. Concentrations of SAA increased from birth to d 10 and remained elevated on d 28 in both groups; however, this increase on d 10 was substantially higher in calves born to OGsupplemented dams compared with those born from NO dams $(P=0.003$, Figure 1a). Overall, calves born from HT dams had higher concentrations of SAA $(P$ $=0.016$, Figure 1a). Concentrations of HPT increased from birth to d 10 and decreased to basal levels on d 28 $(P<0.01)$. Calves born from dams supplemented with OG during late gestation had higher HPT compared with those born from nonsupplemented dams (signifi- cant effect of OG and day, $P<0.04$; Figure 1b). Calves born to CLOG dams tended to have higher levels of HPT compared with CLNO calves $(P=0.09)$.

\section{Neutrophil Function}

The functional capacity of neutrophils was assessed by evaluating their phagocytic and oxidative burst activities. The number of reactive oxygen metabolites produced by circulating neutrophils was affected by the interaction between treatments and day $(P=$ 0.004). Specifically, on d 28 in CLNO calves and on d 10 for HTOG calves, the reactive oxygen metabolites were lower compared with other groups. In contrast, reactive oxygen metabolites did not change over time in HTNO calves and were higher in CLOG on d 10 (Figure 2a). The percentage of neutrophils undergoing oxidative burst was affected by the interaction between treatments and day $(P=0.01)$. The percentage of neutrophils undergoing oxidative burst tended to decrease over time in calves born from CLNO dams, increased in calves born from HTNO dams (reaching the highest percentage on d 28), and was elevated in CLOG dams only on d 10 (Figure 2b). Additionally, calves born to CLOG dams had an elevated percentage of neutrophils undergoing phagocytosis, particularly on d 10 after calving $(P=0.02$, interaction between treatment and day; Figure 2c). 


\section{Blood Hematology}

All calves had more circulating white blood cells (leukocytes) and fewer red blood cells after maternal colostrum consumption (day effect, $P<0.001$; Table 2 ). Circulating hematocrit (erythrocyte ratio of total blood volume), hemoglobin, and platelets also decreased after colostrum consumption for all calves (day effect, $P<0.001$ ). However, calves born from OG cows had greater red blood cell counts, higher hematocrit, and higher hemoglobin compared with those born from
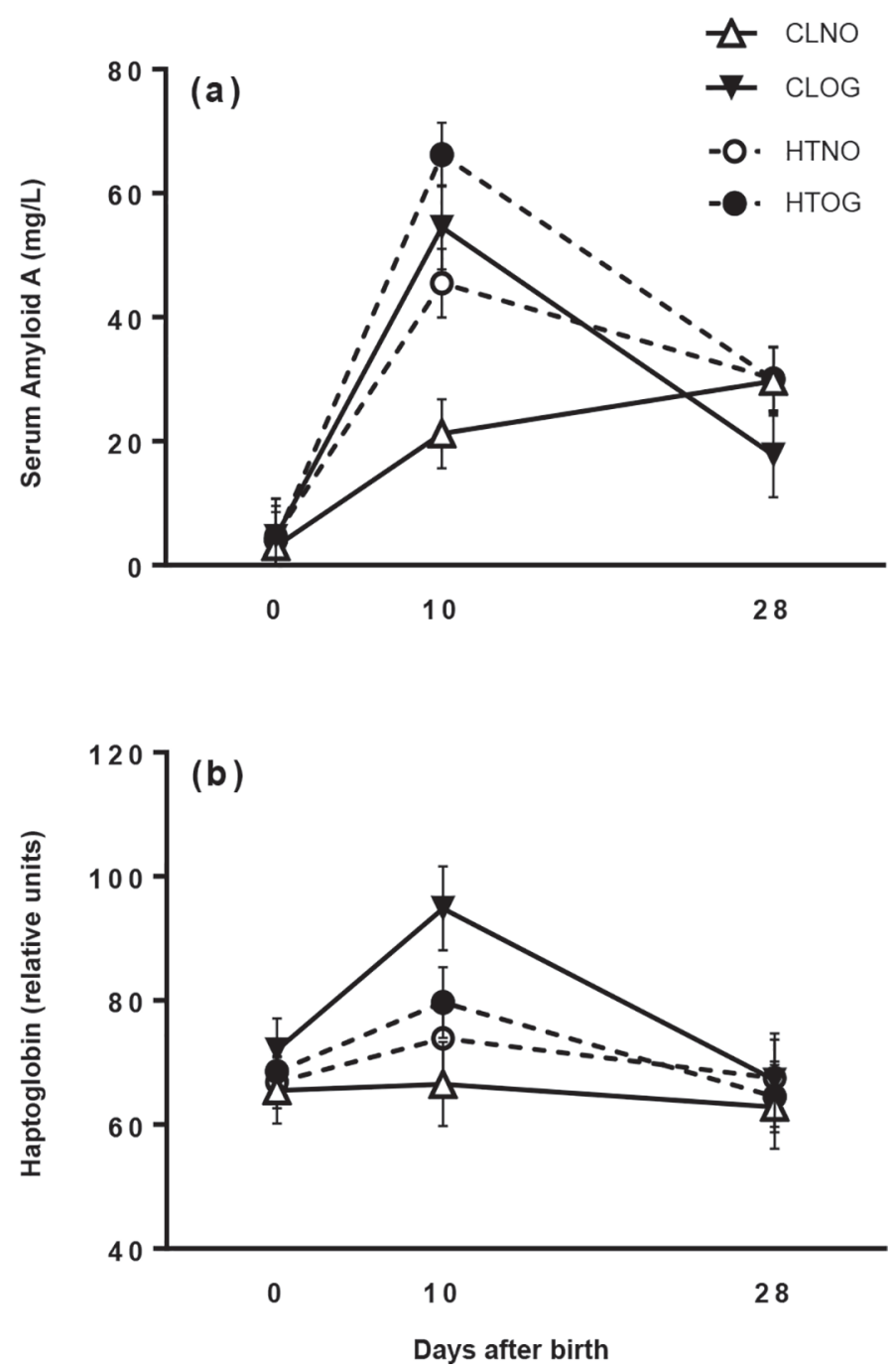

Figure 1. Circulating acute phase proteins from calves born to cooled (CL) or heat-stressed (HT) cows supplemented with OmniGenAF (OG; $56 \mathrm{~g} / \mathrm{d}$, Phibro Animal Health Corporation, Teaneck, NJ) or not supplemented (NO) during late gestation (dry period, approximately $46 \mathrm{~d}$ ). Measurements of (a) serum amyloid A (significant effect of HT and interaction between OG and day, $P<0.016$ ) and (b) haptoglobin (significant effect of $\mathrm{OG}$ and day, $P<0.04$ ). Blood samples were collected at birth (0) and on d 10 and 28 after calving. Graphs describe the interaction between treatments and days. Data are presented as LSM \pm SEM
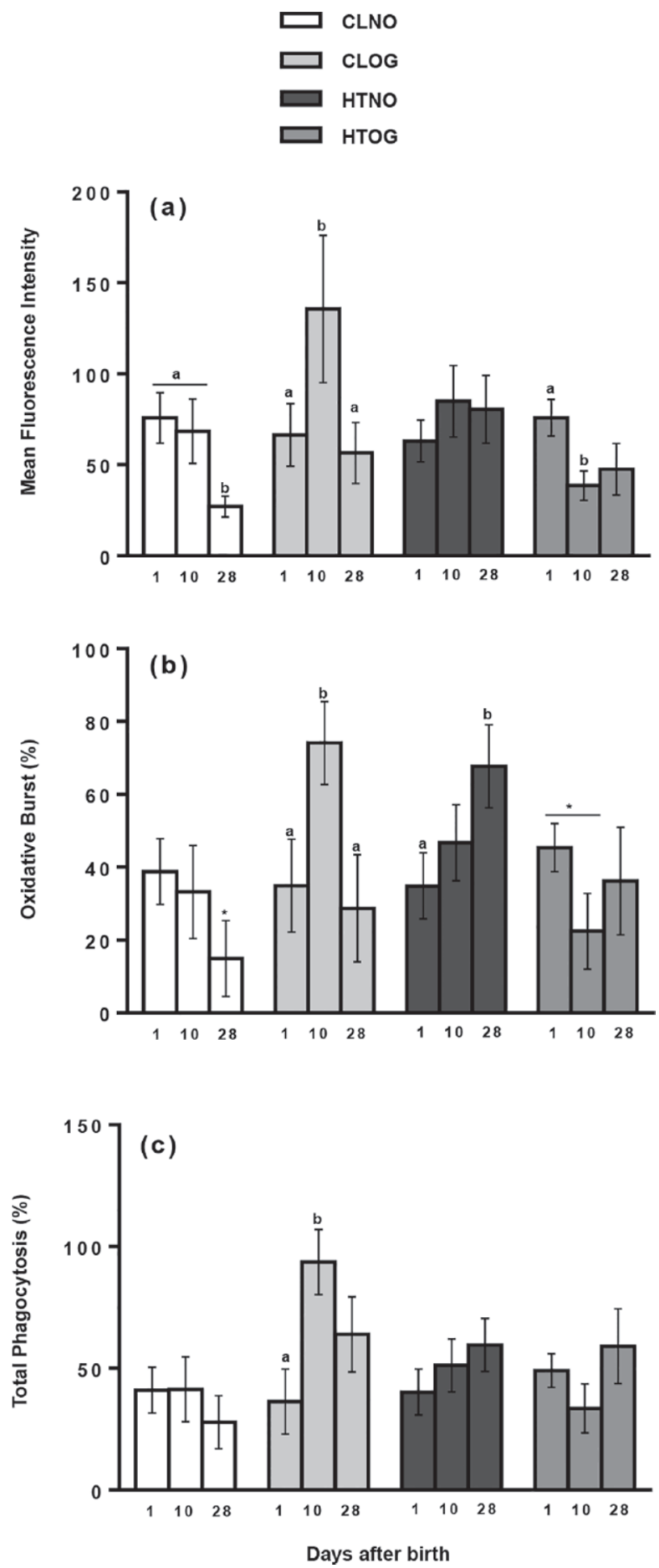

Figure 2. Phagocytic and oxidative burst activities of calves born to cows that were cooled (CL) or heat-stressed (HT) during late gestation (dry period, approximately $46 \mathrm{~d}$ ) and supplemented with OmniGen-AF (OG; 56 g/d, Phibro Animal Health Corporation, Teaneck, NJ) or not supplemented (NO). Blood samples were collected at $24 \mathrm{~h}$ after birth (d 1), d 10 and 28 after calving. (a) Mean amount of reactive oxygen metabolites produced by neutrophils, and (b) percentage of neutrophils undergoing oxidative burst and (c) phagocytosis. Graphs describe the interaction between treatments and day, data are presented as LSM \pm SEM; different letters indicate statistical difference $(P \leq 0.05),{ }^{*} 0.05<P \leq 0.10$. 
Table 2. Hematology analysis of calves born to cool (CL) or heat-stress (HT) cows supplemented (OG) or not supplemented (NO) with OmniGen-AF (Phibro Animal Health Corporation, Teaneck, NJ) during late gestation (dry period, approximately 46 d) ${ }^{1}$

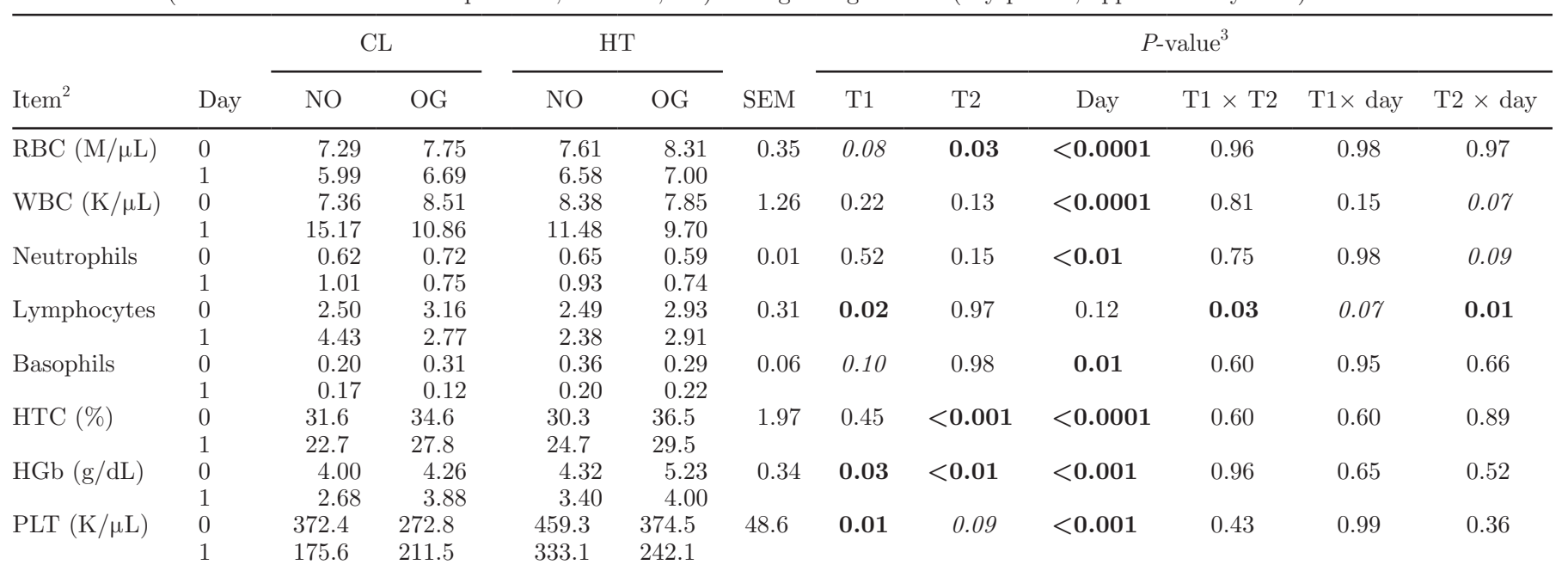

${ }^{1}$ Blood samples were collected immediately after birth (before colostrum feeding) and at $24 \mathrm{~h}$ after birth (d 0 and 1 ). Data are presented as mean \pm SEM; $P \leq 0.05$ indicates statistical difference (in bold), and $0.05<P$-value $<0.10$ indicates a tendency (in italics).

${ }^{2} \mathrm{RBC}=$ red blood cell counts, total number of erythrocytes; WBC $=$ white blood cell count, total number of leukocytes; HTC $=$ hematocrit, erythrocyte ratio of total blood volume; $\mathrm{HGb}=$ hemoglobin; PLT $=$ platelet count (units: $\mathrm{M}, \times 10^{3} / \mu \mathrm{L} ; \mathrm{K}, \times 10^{6} / \mu \mathrm{L}$ ).

${ }^{3} \mathrm{~T} 1=$ heifers born from dams that were heat stressed (HT) or cooled (CL) during late gestation; T2 = heifers born from dams supplemented with OmniGen-AF $(56 \mathrm{~g} / \mathrm{d})$ or not supplemented during late gestation ( $\mathrm{n}=6-8$ per treatment).

nonsupplemented dams (OG effect, $P<0.03$ ). Calves born from HT dams had more platelets and more circulating hemoglobin compared with those born to CL dams during late gestation $(P<0.03)$, but we observed no interaction between treatments. Calves born to HT dams had lower lymphocytes $(P=0.02)$ and tended to have higher basophils $(P=0.10)$ than those born to CL dams. At birth, calves born to OG-supplemented dams had more lymphocytes than calves born to nonsupplemented dams (interaction $\mathrm{OG}$ with day, $P=0.01$ ).

\section{DISCUSSION}

Short- and long-term effects on the physiology and immune function of calves gestated under conditions of heat stress have been demonstrated (Tao et al., 2012a, 2014; Monteiro et al., 2014). Supplementation of OG increases milk production and modulates immune function in both thermo-neutral and heat-stressed cows (Hall et al., 2014; Brandão et al., 2016; Fabris et al., 2017); however, the effects on calves born to OGsupplemented dams had not yet been tested. Herein, we describe the effects of supplementing late-gestation heat-stressed or actively cooled dams with or without OG on the growth and innate immunity of the calves gestated under these conditions.

Despite similarities in THI between heat and cooled pen environments, rectal temperature and respiration rate were higher in HT cows throughout the dry pe- riod, confirming achievement of an effective heat stress treatment in pregnant HT cows. Although OG supplementation during the dry period did not affect dam rectal temperature at calving, calves born to HTNO cows tended to have higher rectal temperatures at birth relative to the other treatment groups, suggesting that both maternal OG supplementation and active cooling of dams during late gestation were effective in reducing postnatal body temperature of the calves. Effects of maternal OG supplementation on calf body temperature dissipated by d 10 postpartum, as in utero HT calves continued to have higher body temperature than in utero CL calves, regardless of maternal OG supplementation. These results confirm previous reports of persistent effects of in utero thermal stress on calf thermoregulatory capacity (Tao et al., 2012a; Johnson et al., 2015; Monteiro et al., 2016b; Laporta et al., 2017), which appears to confer an adaptive advantage for in utero HT calves when exposed to acute heat stress later in life (Ahmed et al., 2015).

Calves exposed to in utero heat stress were lighter at birth and tended to have lower ADG from birth to weaning relative to in utero CL calves. Additionally, OG-supplemented cows tended to give birth to heavier calves relative to NO cows, but their growth rates during the preweaning period were similar. Differences between in utero HT and CL in birth weight are consistent with previous studies using a similar heat stress model (do Amaral et al., 2009; Tao et al., 2012a, 
2014; Monteiro et al., 2014) and may be attributed to a truncated gestation length, reduced maternal feed intake, or placental insufficiency associated with heat stress in dairy cows (Collier et al., 1982; Tao et al., 2011, 2012a; Fabris et al., 2017). Notably, the gestation length was not truncated in the OG dams, and they also had reduced respiration rates during the dry period and produced heavier calves compared with HT dams. It is possible that OG helps to ameliorate the gestational stress caused by heat stress.

Maternal cooling and OG supplementation during late gestation affected passive and innate immunity of calves from birth to weaning. First, apparent efficiency of $\operatorname{IgG}$ absorption was greater in calves born to CL dams than those born to HT dams, corroborating results of studies using a similar experimental HT design (Tao et al., 2012a; Monteiro et al., 2014; Laporta et al., 2017). This is not attributed to differences in colostral IgG concentration, as HT dams had a greater concentration of IgG in colostrum than CL dams, indicating that in utero HT calves have a lower capacity to absorb IgG than in utero CL calves. Second, the acute phase response was altered by our experimental treatments. The acute phase response is a systemic reaction to stress, tissue injury, or infection, resulting in leukocytosis, hormone release, and production of acute phase proteins to stymie tissue damage and induce tissue repair processes (Baumann and Gauldie; 1994, Koj, 1996; Ceciliani et al., 2012). As such, the acute phase response is an integral innate immune process. In our study, concentrations of SAA and HPT, 2 primary bovine acute phase proteins, were both higher in the circulation of calves born to OG dams relative to calves born to $\mathrm{NO}$ dams, particularly at $10 \mathrm{~d}$ of age. Similarly, LPS infusion induced a greater acute phase response in mature dairy and beef cattle supplemented with OG relative to nonsupplemented controls, suggesting greater capacity of supplemented cows to mount an innate immune response (Burdick Sanchez et al., 2014; Brandão et al., 2016). The acute phase response is not without cost, as energy and AA are required for production of acute phase proteins, and often these resources come from tissue catabolism (Ceciliani et al., 2012). However, the transient nature of the acute phase response in the calves, and the similar average daily weight gain between $\mathrm{OG}$ and $\mathrm{NO}$ calves, indicate that the greater acute phase response of OG calves had negligible effects on calf growth.

In utero HT calves had similar concentrations of plasma HPT but significantly higher SAA concentration throughout the preweaning period relative to in utero CL calves. In accordance, Tao et al. (2013) found that cows heat-stressed during late gestation have elevated mRNA expression of tumor necrosis factor $\alpha$ and IL-8 in PBMC, 2 proinflammatory cytokines that stimulate production of positive acute phase proteins (Petersen et al., 2004). However, despite enhanced cytokine and acute phase protein production, immune responses of heat-stressed cows to a pathogen may be dampened. For instance, stimulating lymphocytes in vitro with a mitogen lowered cytokine production in lymphocytes isolated from heat-stressed cows relative to those from cooled cows (do Amaral et al., 2010).

It is intriguing to speculate on potential mechanisms through which maternal heat stress or dietary OG supplementation of the dam alters the acute phase response of the postnatal calf. In neonates, the acute phase response can be activated by maternally derived acute phase proteins or cytokines transferred through colostrum. In dairy calves, SAA is not transferred in colostrum and, although it is unknown if HPT transfer through colostrum occurs in calves, colostral HPT is absorbed across the neonatal intestine in piglets (Orro et al., 2008; Hiss-Pesch et al., 2011). Moreover, colostrum contains numerous cytokines that can stimulate acute phase protein production in the neonate (Hagiwara et al., 2000). The similar SAA and HPT levels among calves in all treatment groups before, but not following, colostrum feeding support a colostral pathway of acute phase response induction in bovine calves.

Measures of neutrophil function support our hypothesis that maternal OG supplementation in combination with cooling would benefit calf immune status. Although calves from all 4 treatment groups had similar circulating neutrophil concentrations at birth and $\mathrm{d} 1$ of age, neutrophil function was enhanced in CLOG calves. Specifically, calves born to dams that were actively cooled during the dry period and $\mathrm{OG}$ had a greater proportion of neutrophils undergoing phagocytosis and oxidative burst and the neutrophils had elevated production of reactive oxygen metabolites, particularly at d 10 of age. Neutrophils migrate to and infiltrate sites of infection to phagocytize and kill bacteria, and in the process undergo respiratory burst and degranulation, which produces reactive oxygen metabolites (Paape et al., 2003). Leukocytosis is aided by a protein, Lselectin, on neutrophil cell surfaces that allows the cells to interact with vascular endothelial cells and migrate to the infected site (Weber et al., 2004). L-Selectin protein and mRNA expression as well as reactive oxygen metabolite production by neutrophils was also greater in Holsteins and immunosuppressed sheep supplemented with OG (Wang et al., 2007; Ryman et al., 2013). Collectively these studies are indicative of the benefits of OG supplementation on neutrophil migration and function.

Erythrocyte, total leukocyte, neutrophil, and basophil counts were similar between HT and CL calves in 
our study, but hemoglobin concentration was higher for in utero HT calves relative to in utero CL calves. Our results contrast with 2 studies of heat stress in pigs and sheep that found either no differences in hemoglobin levels between heat-stressed or thermoneutral animals or lower hemoglobin in heat-stressed individuals (Morrow-Tesch et al., 1994; Srikandakumar et al., 2003); however, hemoglobin in those studies were measured in thermally stressed adults. The higher concentration of hemoglobin in HT calves in our study could reflect a hypoxic intrauterine environment, as demonstrated in human infants (Cook et al., 1957). Furthermore, the higher hemoglobin concentration of HT calves is not likely a result of hemoconcentration or dehydration, as hematocrit and erythrocyte counts were similar between treatment groups. Additionally, in our study, platelet count was higher in $\mathrm{HT}$ calves relative to CL calves. This is not surprising given the higher circulating concentration of acute phase protein in HT calves. Besides their role in blood clotting, platelets are involved in the inflammatory response, releasing molecules that mediate numerous inflammatory reactions from changes in blood vessel diameter to neutrophil chemotaxis (Klinger, 1997). Calves born to OG dams had more lymphocytes at birth, suggesting greater cellmediated immunity, which is in congruence with previous studies showing greater lymphocyte concentrations in OG-supplemented livestock (Wang et al., 2007, 2009). In addition, OG calves had a higher erythrocyte count, hematocrit, and hemoglobin levels. Haptoglobin, which was also elevated in the plasma of our OG calves, forms complexes with free hemoglobin in circulation to prevent oxidative damage to hemoglobin and to limit iron availability for bacterial proliferation (Ceciliani et al., 2012).

Herein we demonstrated the benefits of OmniGen-AF supplementation and active cooling of cows during late gestation on their neonates, including a more responsive immune system through increased neutrophil function, acute phase protein production, and changes in immunologically important blood cell counts. Further research on the specific mechanisms through which this commercial feed additive might help improve innate and cell-mediated immunity as well as the long-term effects of maternal supplementation on calf health and productivity is warranted.

\section{ACKNOWLEDGMENTS}

The authors thank the staff of the Dairy Unit of University of Florida for animal care and data collection. This project was supported by Phibro Animal Health Corporation (Teaneck, NJ).

\section{REFERENCES}

Ahmed, B. M., U. Younas, T. Asar, S. Dikman, P. J. Hansen, and G. E. Dahl. 2015. Cows exposed to heat stress in utero exhibit improved thermal tolerance. J. Dairy Sci. 98(Suppl.2):862 (Abstr).

Baumann, H., and J. Gauldie. 1994. The acute phase response. Immunol. Today 15:74-80.

Brandão, A. P., R. F. Cooke, F. N. Corrá, M. B. Piccolo, R. Gennari, T. Leiva, and J. L. M. Vasconcelos. 2016. Physiologic, health, and production responses of dairy cows to supplemented with an immunomodulatory feed ingredient during the transition period. J. Dairy Sci. 99:5562-5572.

Branson, J. A., D. J. McLean, N. E. Forsberg, and G. Bobe. 2016 Yeast-containing feed additive alters gene expression profiles associated with innate immunity in whole blood of a rodent model. Innate Immun. 22:249-256.

Burdick Sanchez, N. C., J. O. Buntyn, J. A. Carroll, T. Wistuba, K. DeHann, S. E. Sieren, S. J. Jones, and T. B. Schmidt. 2014 Enhancement of the acute phase response to lipopolysaccharide in feedlot steers supplemented with OmniGen-AF. J. Anim. Sci. 92(Suppl.2):37-38. (Abstr).

Ceciliani, F., J. J. Ceron, P. D. Eckersall, and H. Sauerwein. 2012 Acute phase proteins in ruminants. J. Proteomics 75:4207-4231.

Collier, R. J., S. G. Doelger, H. H. Head, W. W. Thatcher, and C. J. Wilcox. 1982. Effects of heat stress during pregnancy on maternal hormone concentrations, calf birth weight and postpartum milk yield of Holstein cows. J. Anim. Sci. 54:309-319.

Cook, C. D., H. R. Brodie, and D. W. Allen. 1957. Measurement of fetal hemoglobin in newborn infants: correlation with gestational age and intrauterine hypoxia. Pediatrics 20:272-278.

Cooke, R. F., and J. D. Arthington. 2013. Concentrations of haptoglobin in bovine plasma determined by ELISA or a colorimetric method based on peroxidase activity. Anim. Physiol. Anim. Nutr. (Berl.) 97:531-536.

Dahl, G. E., S. Tao, and A. P. A. Monteiro. 2016. Effects of lategestation heat stress on immunity and performance of calves. J. Dairy Sci. 99:3193-3198.

Dikmen, S., E. Alava, E. Pontes, J. M. Fear, B. Y. Dikmen, T. A. Olson, and P. J. Hansen. 2008. Differences in thermoregulatory ability between slick-haired and wild-type lactating Holstein cows in response to acute heat stress. J. Dairy Sci. 91:3395-3402.

do Amaral, B. C., E. E. Connor, S. Tao, J. Hayen, J. Bubolz, and G. E. Dahl. 2009. Heat-stress abatement during the dry period: does cooling improve transition into lactation? J. Dairy Sci. 92:59885999.

do Amaral, B. C., E. E. Connor, S. Tao, J. Hayen, J. Bubolz, and G. E. Dahl. 2010. Heat stress abatement during the dry period influences prolactin signaling in lymphocytes. Domest. Anim. Endocrinol. 38:38-45.

do Amaral, B. C., E. E. Connor, S. Tao, J. Hayen, J. W. Bubolz, and G. E. Dahl. 2011. Heat stress abatement during the dry peirod influences metabolic gene expression and improves immune status in the transition period of dairy cows. J. Dairy Sci. 94:86-96.

Donovan, A., C. Risco, F. Maunsell, J. Lemmermen, and E. Diepersloot. 2011. IFAS Dairy Unit standard operating procedures. University of Florida, Gainesville, FL. Accessed Apr. 27, 2017. http://animal. ifas.ufl.edu/facilities/du/FloridaDairyUnitSOP11-07-01.pdf.

Fabris, T. F., J. Laporta, F. N. Corra, Y. M. Torres, D. J. Kirk, D. J. McLean, J. D. Chapman, and G. E. Dahl. 2017. Effect of nutritional immunomodulation and heat stress during the dry period on subsequent performance of cows. J. Dairy Sci. In press.

Guo, J.-R., A. P. A. Monteiro, X.-S. Weng, B. M. Ahmed, J. Laporta, M. J. Hayen, G. E. Dahl, J. K. Bernard, and S. Tao. 2016. Effect of maternal heat stress in late gestation on blood hormones and metabolites of newborn calves. J. Dairy Sci. 99:6804-6807.

Hagiwara, K., S. Kataoka, H. Yamanaka, R. Kirisawa, and H. Iwai. 2000. Detection of cytokines in bovine colostrum. Vet. Immunol. Immunopathol. 76:183-190.

Hall, L. W., F. A. Rivera, F. Villar, J. D. Chapman, N. M. Long, and R. J. Collier. 2014. Evaluation of OmniGen-AF in lactating heatstressed Holstein cows. Pages 16-26 in Proc. 25th Annual Florida 
Ruminant Nutrition Symposium. Department of Animal Sciences, University of Florida.

Hiss-Pesch, S., F. Daniel, S. Dunkleberg-Denk, M. Mielenz, and H. Sauerwein. 2011. Transfer of maternal haptoglobin to suckling piglets. Vet. Immunol. Immunopathol. 144:104-110.

Johnson, J. S., M. Abuajamieh, V. M. Sanz Fernandez, J. T. Seibert, S. K. Stoakes, A. F. Keating, J. W. Ross, J. T. Selsby, R. P. Rhoads, and L. H. Baumgard. 2015. (b). The impact of in utero heat stress and nutrient restriction on progeny body composition. J. Therm. Biol. 53:143-150.

Klinger, M. H. F. 1997. Platelets and inflammation. Anat. Embryol. (Berl.) 196:1-11.

Koj, A. 1996. Initiation of acute phase response and synthesis of cytokines. Biochim. Biophys. Acta 1317:84-94.

Laporta, J., T. F. Fabris, A. L. Skibiel, J. L. Powell, M. J. Hayen, K. Horvath, E. K. Miller-Cushon, and G. E. Dahl. 2017. In utero exposure to heat stress during late gestation has prolonged effects on the activity patterns and growth of dairy calves. J. Dairy Sci. 100:2976-2984.

Martinez, N., C. A. Risco, F. S. Lima, R. S. Bisinotto, L. F. Greco, E. S. Ribeiro, F. Maunsell, K. Galvão, and J. E. Santos. 2012. Evaluation of peripartal calcium status, energetic profile, and neutrophil function in dairy cows at low or high risk of developing uterine disease. J. Dairy Sci. 95:7158-7172.

McGuirk, S. M. 2008. Disease management of dairy calves and heifers. Vet. Clin. North Am. Food Anim. Pract. 24:139-153.

Monteiro, A. P. A., J.-R. Guo, X.-S. Weng, B. M. Ahmed, M. J. Hayen, G. E. Dahl, J. K. Bernard, and S. Tao. 2016a. Effect of maternal heat stress during the dry period on growth and metabolism of calves. J. Dairy Sci. 99:3896-3907.

Monteiro, A. P. A., S. Tao, I. M. Thompson, and G. E. Dahl. 2014 Effect of heat stress during late gestation on immune function and growth performance of calves: isolation of altered colostral and calf factors. J. Dairy Sci. 97:6426-6439.

Monteiro, A. P. A., S. Tao, I. M. T. Thompson, and G. E. Dahl. 2016b. In utero heat stress decreases calf survival and performance through the first lactation. J. Dairy Sci. 99:8443-8450.

Morrow-Tesch, J. L., J. J. McGlone, and J. L. Salak-Johnson. 1994 Heat and social stress effects on pig immune measures. J. Anim. Sci. 72:2599-2609.

Orro, T., S. Jacobsen, J.-P. LePage, T. Niewold, S. Alasuutari, and T. Soveri. 2008. Temporal changes in serum concentrations of acute phase proteins in newborn dairy calves. Vet. J. 176:182-187.

Paape, M. J., D. D. Bannerman, X. Zhao, and J.-W. Lee. 2003. The bovine neutrophil: structure and function in blood and milk. Vet. Res. 34:597-627.

Petersen, H. H., J. P. Nielsen, and P. M. H. Heegaard. 2004. Application of acute phase protein measurements in veterinary clinical chemistry. Vet. Res. 35:163-187.
Quigley, J. D., III, and J. J. Drewry. 1998. Nutrient and immunity transfer from cow to calf pre- and postcalving. J. Dairy Sci. 81:2779-2790.

Rowson, A. D., Y.-Q. Wang, E. Aalseth, N. E. Forsberg, and S. B. Puntenney. 2011. Effects of immunomodulatory feed additive on the development of mastitis in a mouse infection model using four bovin-origin isolates. Animal 5:220-229.

Ryman, V. E., S. C. Nickerson, F. M. Kautz, D. J. Hurley, L. O. Ely, Y. Q. Wang, and N. E. Forsberg. 2013. Effect of dietary supplementation on the antimicrobial acitivity of blood leukocytes isolated from Holstein heifers. Res. Vet. Sci. 95:969-974.

Srikandakumar, A., E. H. Johnson, and O. Mahgoub. 2003. Effect of heat stress on respiratory rate, rectal temperature and blood chemistry in Omani and Australian Merino sheep. Small Rumin. Res. 49:193-198.

Tao, S., J. W. Bubolz, B. C. do Amaral, I. M. Thompson, M. J. Hayen, S. E. Johnson, and G. E. Dahl. 2011. Effect of heat stress during the dry period on mammary gland development. J. Dairy Sci. 94:5976-5986.

Tao, S., E. E. Connor, J. W. Bubolz, I. M. Thompson, B. C. do Amaral, M. J. Hayen, and G. E. Dahl. 2013. Effect of heat stress during the dry period on gene expression in mammary tissue and peripheral blood mononuclear cells. J. Dairy Sci. 96:378-383.

Tao, S., and G. E. Dahl. 2013. Heat stress effects during late gestation on dry cows and their calves. J. Dairy Sci. 96:4079-4093.

Tao, S., A. P. A. Monteiro, M. J. Hayen, and G. E. Dahl. 2014. Maternal heat stress during the dry period alters postnatal whole-body insulin response of calves. J. Dairy Sci. 97:897-901.

Tao, S., A. P. A. Monteiro, I. M. Thompson, M. J. Hayen, and G. E. Dahl. 2012a. Effect of late-gestation maternal heat stress on growth and immune function of dairy calves. J. Dairy Sci. 95:71287136

Tao, S., I. M. Thompson, A. P. Monteiro, M. J. Hayen, L. J. Young, and G. E. Dahl. 2012b. Effect of cooling heat-stressed dairy cows during the dry period on insulin response. J. Dairy Sci. 95:50355046 .

Wang, Y., S. B. Puntenney, J. L. Burton, and N. E. Forsberg. 2007. Ability of commercial feed additive to modulate expression of innate immunity in sheep immunosuppressed with dexamethasone. Animal 1:945-951.

Wang, Y.-Q., S. B. Puntenney, J. L. Burton, and N. E. Forsberg. 2009. Use of gene profiling to evaluate the effects of feed additive on immune function in periparturient dairy cattle. J. Anim. Physiol. Anim. Nutr. (Berl.) 93:66-75.

Weber, P. S., T. Toelboell, L. C. Chang, J. D. Tirrell, P. M. Saama, G. W. Smith, and J. L. Burton. 2004. Mechanisms of glucocorticoid-induced down-regulation of neutrophil L-selectin in cattle: evidence for effects at the gene-expression level and primarily on blood neutrophils. J. Leukoc. Biol. 75:815-827. 